\title{
HOMOGENISING AND SEGMENTING HYPERSPECTRAL IMAGES OF PLANTS AND TESTING CHEMICALS IN A HIGH-THROUGHPUT PLANT PHENOTYPING SETUP
}

\author{
Puneet Mishra $^{1 *}$, Martin Schmuck $^{2}$, Sina Roth ${ }^{3}$, Andreas Nicol ${ }^{2}$, Alison Nordon ${ }^{1}$ \\ ${ }^{1}$ WestCHEM, Department of Pure and Applied Chemistry and Centre for Process Analytics and Control \\ Technology, University of Strathclyde, Glasgow, G1 1XL, United Kingdom \\ ${ }^{2}$ Bayer AG, Engineering \& Technology, Screening Technology, Building E 41, 51368 Leverkusen, \\ Germany \\ ${ }^{3}$ Bayer Crop Science, Industriepark Höchst, Building H 872, 107, 65926 Frankfurt, Germany
}

\begin{abstract}
Use of hyperspectral imaging (HSI) for automated characterisation of plants in a high-throughput plant phenotyping setup (HTPPS) is a challenging task. A challenge arises when the same plant is being monitored automatically during the experiment as it might not be in the same orientation as it was imaged last time. Such changes in orientation result in variations in illumination, which affects the signals recorded by the HSI setup. In addition, there are challenges with the use of threshold-based segmentation approaches such as normalised difference vegetation index (NDVI) for distinguishing between old and dead leaves, which might be observed in the later stages of experiments, from the soil background. Therefore, the potential of spectral normalisation for homogenising HS images and the use of supervised spectral set for plant segmentation is presented. Further, the effects of testing chemicals on plants were visualised using PCA of the HS images.
\end{abstract}

Index Terms - visible-near infrared, standard normal variate, high-throughput plant phenotyping setup, automated

\section{INTRODUCTION}

HSI integrated to a HTPPS is emerging as a potential tool for early detection of stress-related symptoms in plant phenotyping related studies [1]. A major benefit of HSI compared to other imaging sensors is that it provides complementary spectroscopic and imaging information [2]. The visible region provides access to the pigments' concentration and the near-infrared region provides information related to the moisture and internal structure of leaves [3]. However, implementing HSI for automated characterisation of plants in a HTPPS is a challenging task; different issues arise starting from handling and imaging plants to the processing of the huge volume of data generated by phenotyping experiments.
A challenge with the monitoring of plants in an automated setup arises from the mechanical movement of the plant to the imaging part of the setup; the orientation of the plant with respect to the camera varies. Such a change in orientation causes differences in the illumination of the surface of the plant leaves, which gives rise to differences in the spectra. These differences can bias the modelling and therefore need to be homogenised.

Another challenge comes from segmentation of images where threshold-based methods such as those based on NDVI estimations are commonly used in close range HSI [1]. NDVI threshold-based segmentation is sufficient when the plant is green and very distinct compared to the background. However, in the later stages of experiments when plants are old and leaves start to turn brown or in experiments testing chemicals or other stress inducers where part of the plant starts to die, then selection of the NDVI threshold is a difficult task. In such cases, the NDVI for the background soil is similar to that for the old and dead leaves of the plant, and hence NDVI threshold-based segmentation will remove the contributions from the old and dead plants together with the soil from the image. However, retention of data from these old plants is important for modelling to capture the complete image change over time.

A challenge also exists from a data visualisation point of view as the HS image typically consist of hundreds of bands and information can be extracted directly from the experiments. Visualising individual wavelengths or calculation of indices can provide an image with enhanced contrast. However, as the indices are usually calculated independently for each image, it is difficult to deduce how they relate to the next time point or other images within the same experiment. In such a case, identifying the main sources of variability in HS images and then using this information to understand the evolution of the system can be a solution. Principal component analysis (PCA) can support this task as the extracted principal components (PCs) can explain the sources of variability during experiments, which 
can subsequently be used to generate contrast maps supporting visualisation of 3D HSI cubes as 2D score maps. The objective of this work is to show the potential of a spectral normalisation technique, standard normal variate (SNV), to homogenise HS images acquired of plants in a HTPPS. Further, the study presents a spectral similaritybased segmentation approach utilising a supervised set. Finally, the study presents an application of PCA for visualising the testing of chemicals on plants in a phenotype study.

\section{MATERIAL AND METHODS}

\subsection{Image acquisition}

Images were recorded with a HTPPS at Bayer Crop Science, Frankfurt, Germany. To record the images, the setup utilises a push broom line-scan hyperspectral camera from Headwall Photonics, Massachusetts, USA. The camera recorded the image with a spectral range of $400-1000 \mathrm{~nm}$ recording 270 spectral bands with 640 spatial pixels. The number of spatial pixels recorded in each scan was $1600 \times$ 1600. The illumination was provided with two halogen bulbs aligned at $45^{\circ}$ to the field of view (FOV) of the camera. The plant was automatically brought to the camera and imaging of the plant was performed by keeping the plant still at the FOV and moving the camera, using a motorised stage, to record the plant and the white reference standard (spectralon). For the evaluation of the homogenisation and segmentation methodologies, images were acquired of two plants, of the same type, at different growth stages with the plants measured at four different orientations relative to the camera. The images acquired for chemical testing were of 7 plants where 1 plant was untreated and 6 were treated with different chemicals.

\subsection{Samples description}

\subsection{Data processing}

\subsubsection{Radiometric calibration}

Radiometric calibration utilising dark and white reference images was performed for every pixel in the HS image according to equation (1):

$$
I_{R(i, j, k)}=\frac{I_{\text {raw }(i, j, k)}-I_{\operatorname{dark}(i, j, k)}}{I_{\text {white }(i, i, k)}-I_{\operatorname{dark}(i, i, k)}}
$$

where, $I_{R}$ is the calibrated reflectance, $I_{\text {raw }}$ is the raw intensity measured from the test sample, $I_{\text {dark }}$ is the intensity of the dark response, $I_{\text {white }}$ is the intensity of the uniform white reference, and $i$ and $j$ are spatial coordinates and $k$ is the wavelength of the image.

\subsubsection{Smoothing and Normalisation}

The spectral range of the hypercube was reduced to $450-$ $900 \mathrm{~nm}$ to remove the noisy part of the spectrum. Further, a moving window Savitzky-Golay filter [4] (15-point width and second order polynomial) was applied to each pixel of the image to remove random noise, e.g. spikes, from spectra. Further, to reduce light scattering effects arising from inhomogeneity of the leaf surface, the spectra were normalised using the SNV transformation $[1,5]$.

\subsubsection{Segmentation}

The segmentation approach used in this work involved comparing a set of supervised spectra with each pixel in a HS image and then assigning the pixel to a class; with 1 and 0 denoting membership and non-membership of a class, respectively. The spectral similarity was measured utilising the Euclidean Distance (ED) and the pixels were assigned to a class on the basis of the minimum distance to a spectral set. For comparison with the methodologies presented here, NDVI was also calculated for the HS images of plants using equation (2):

$$
\operatorname{NDVI}(i, j)=\frac{I_{R}(i, j, 740)-I_{R}(i, j, 670)}{I_{R}(i . i .740)+I_{R}(i . i .670)}
$$

where, $i$ and $j$ are spatial coordinates and the values of 670 and 740 are wavelengths (in $\mathrm{nm}$ ).

\subsubsection{Principal component analysis}

PCA transforms a set of observations containing correlated variables to observations containing linearly uncorrelated variables defined as principal components (PCs) [6]. The basic consideration used for implementing PCA is that the transformation is performed to retain the maximum amount of variability in the dataset. Furthermore, the extraction of PCs is carried out in such a way that the first PCs retain the maximum amount of variability, and the subsequent PCs contain the highest amount of variation but orthogonal to the variability explained by the PCs previously extracted. In this way, the PCs define a new orthonormal basis set which can be used to transform the data from a high dimension space to the lower space explained by the PCs. In present work, a global PCA was performed over all the images to present explainable differences. PCA was performed by unfolding the $3 \mathrm{D}$ cubes to $2 \mathrm{D}$ matrices and later refolding the scores to generate false color images.

\section{RESULTS}

Figure 1 presents images acquired of the two plants at different growth stages and at four different orientations relative to the HSI camera. Plant 1 was mature compared to 
plant 2 as can be see with the canopy area visually. The red circles in Figure 1 show how the orientation of the plant was tracked. The orientation was denoted $0,90,180$ and $270^{\circ}$.
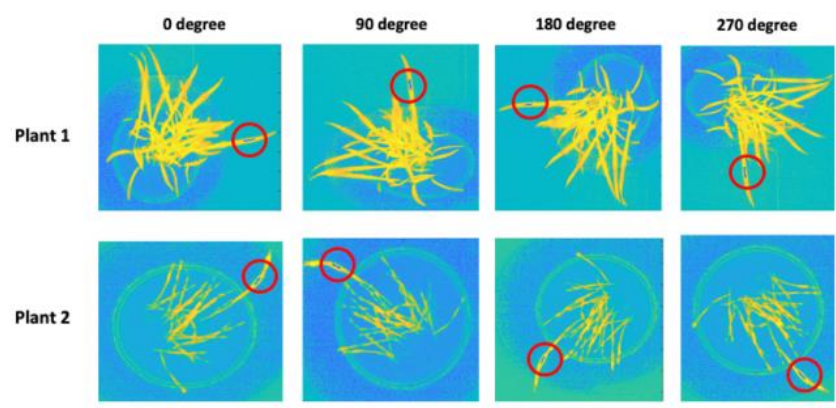

Figure 1: Two plants at different growth stage acquired at different orientations relative to the camera. The red circles were used to track the orientation of the plant relative to the camera.

The mean reflectance spectra (without and with normalisation) extracted from the images of plant 1 and 2 can be seen in Figures 2 and 3, respectively. It can be seen clearly in Figures 2(a) and 3(a) that when the same plant is imaged at different orientations, the reflectance spectra are different owing to variations in the illumination of the plant. However, it can be seen that normalisation using the SNV transformation removes these differences as shown in Figures 2(b) and 3(b).
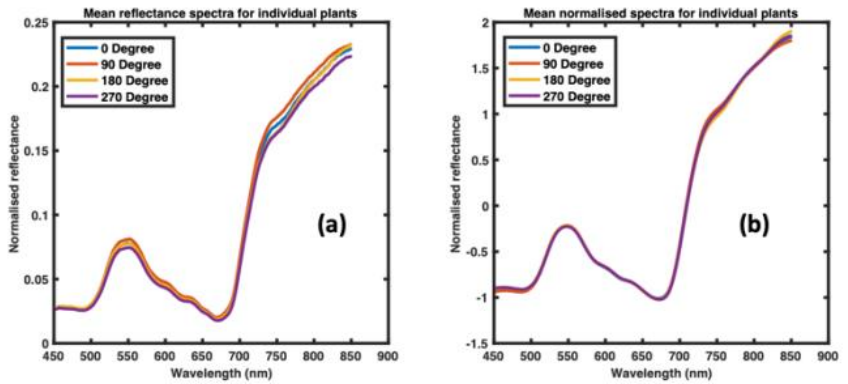

Figure 2: Mean spectra from plant 1 at 4 different orientations. (a) Reflectance spectra and (b) normalised spectra.
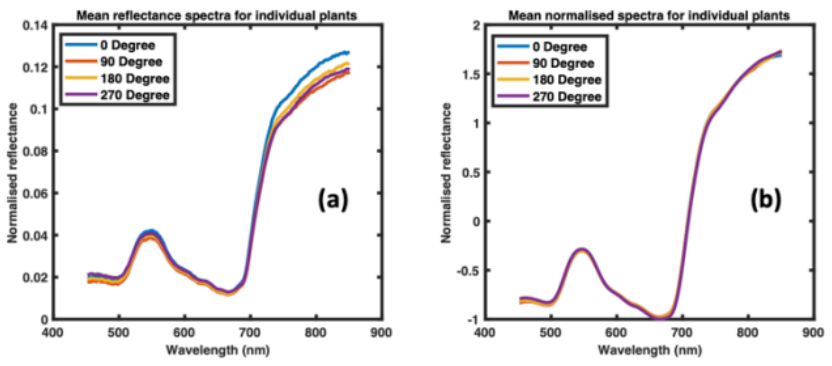

Figure 3: Mean spectra from plant 2 at 4 different orientations. (a) Reflectance spectra and (b) normalised spectra.

Figures 4 and 5 presents the results of segmentation with NDVI and similarity, respectively, based on a supervised spectral dataset. It can be seen that it is difficult to segment the dead leaves using the NDVI (Figure 4), whereas the similarity-based method (Figure 5) provides a better segmentation of the plants. Increasing the NDVI threshold removed the old/dead parts of the plant along with the soil and background, whereas choosing a low NDVI retained features in the image that do not belong to the plants.
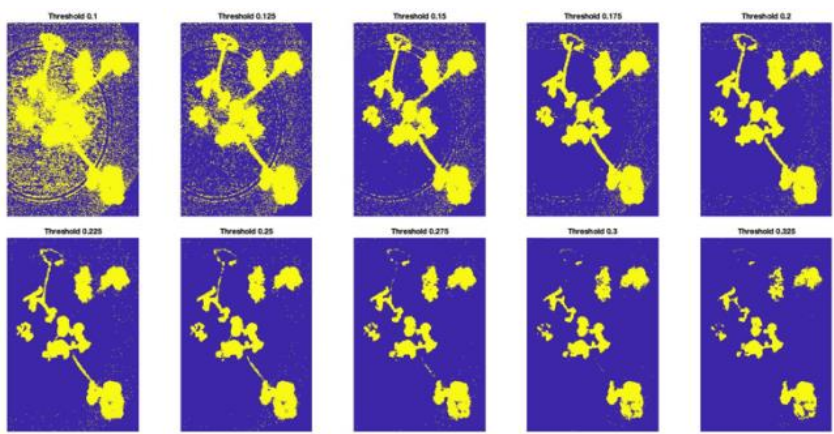

Figure 4: Masks generated from NDVI threshold.

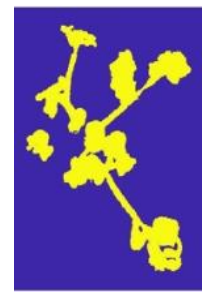

Figure 5: Segmentation performed utilising similarity to a supervised dataset. 


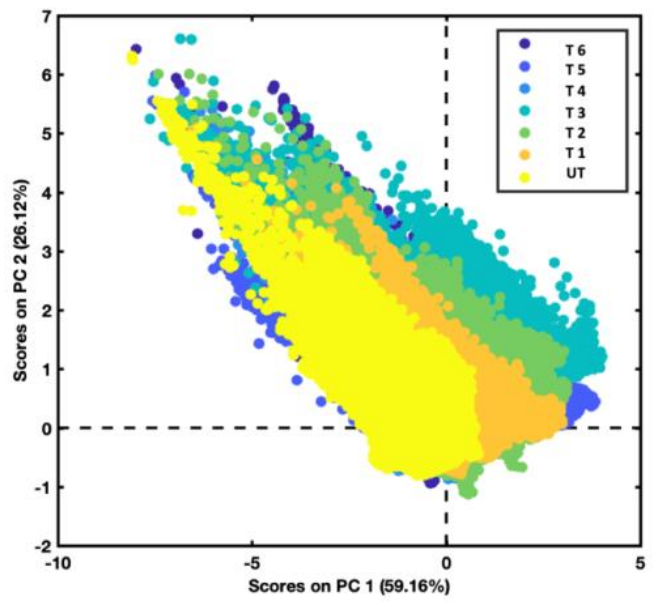

Figure 6: Score 1 vs Score 2 samples used in chemical testing. UT denotes the untreated plant while T1 to T6 denote the six plants that were treated.

Figure 6 presents the results of PCA performed on the image set presented in Figure 7 related to chemical analysis. It can be seen in Figure 6 that the scores of the untreated plant lie mainly in the center of the PC biplot. However, after treatment with the chemical the PC1 scores for the plant increases. The increase in score gives an indication of the potency of the different chemicals, with plants having much higher PC1 scores being more damaged by the treatment of the plant with a chemical (e.g. T3). The score maps obtained from PCA of the HS images are presented in Figure 7.

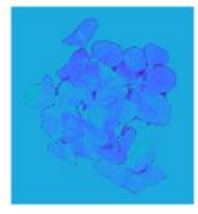

Untreated

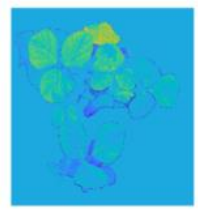

Treatment 4

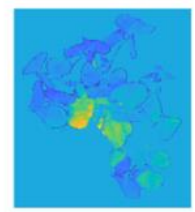

Treatment 1

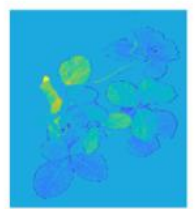

Treatment 5

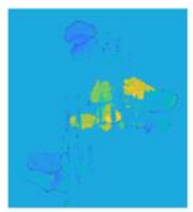

Treatment 2

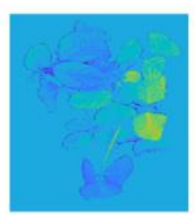

Treatment 6

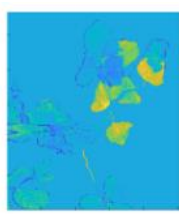

Treatment 3
Figure 7: False colour images, generated from PC1 scores, of the untreated plant and the 6 plants treated with different chemicals.

The scores maps presented in Figure 7 show similar changes caused by the application of chemicals to the plants. It can be seen from Figure 7 that the PC scores for the untreated plant are similar over most of the plant (primarily blue), whereas differences (indicated by the yellow regions) are apparent in those plants treated with chemicals. The brightest yellow region represents the most damaged part of the plant, whereas the greenish yellow region represents intermediate damage. It can be concluded from Figures 6 and 7 that treatment 3 seems to be the most potent, which gives rise to the highest $\mathrm{PC} 1$ scores.

\section{CONCLUSIONS}

In the present work, a HTPPS setup was utilised to perform HSI imaging of plants. The results showed that normalisation can homogenise the HS images, which can eliminate spectral variability arising from differences in illumination that can occur in continuous automated measurements. In addition, it has been shown that plant segmentation utilising a supervised spectral dataset based on similarity is a practical solution for segmenting plants, which have older and/or dead parts. Further, it has been shown that for an initial understanding of the data, variance capturing analysis such as PCA can be used and 3D HS images can be visualised as 2D false colour maps. Future work will include developing metrics to quantify the changes in the PC space which can be used for decision making.

\section{ACKNOWLEDGMENTS}

This work has received funding from the European Union's Horizon 2020 research and innovation program under the Marie Skłodowska-Curie grant agreement number 675251

\section{REFERENCES}

[1] M.S. Mohd Asaari, P. Mishra, S. Mertens, S. Dhondt, D. Inzé, N. Wuyts, P. Scheunders, "Close-range hyperspectral image analysis for the early detection of stress responses in individual plants in a high-throughput phenotyping platform," ISPRS Journal Photogrammetry Remote Sensing, 138 (2018) 121-138.

[2] N. Fahlgren, M.A. Gehan, I. Baxter, "Lights, camera, action: high-throughput plant phenotyping is ready for a close-up," Current Opinion Plant Biology, 24 (2015) 93-99.

[3] P. Mishra, M.S.M. Asaari, A. Herrero-Langreo, S. Lohumi, B. Diezma, P. Scheunders, "Close range hyperspectral imaging of plants: A review," Biosystem Engineering, 164 (2017) 49-67.

[5] A. Savitzky, M.J.E. Golay, "Smoothing and Differentiation of Data by Simplified Least Squares Procedures," Analytical Chemistry, 36 (1964) 1627-1639.

[5] R.J. Barnes, M.S. Dhanoa, S.J. Lister, "Standard Normal Variate Transformation and De-Trending of Near-Infrared Diffuse Reflectance Spectra," Applied Spectroscopy, 43 (1989) 772-777.

[6] S. Wold, K. Esbensen, P. Geladi, "Principal component analysis", Chemometrics and Intelligent Laboratory Systems, 2 (1987) 37-52. 
